\title{
P-Wave Shape Changes Observed in the Surface Electrocardiogram of Subjects with Obstructive Sleep Apnoea
}

\author{
S Boudaoud $^{1}, \mathrm{C}$ Heneghan $^{2}, \mathrm{H} \mathrm{Rix}^{1}, \mathrm{O}^{\text {Meste }^{1}}{ }^{1}, \mathrm{C} \mathrm{O}^{\prime} \mathrm{Brien}^{2}$ \\ ${ }^{1}$ University of Nice-Sophia Antipolis, Lab I3S, France \\ ${ }^{2}$ University College Dublin, School of Electrical, Electronic and Mechanical Engineering, Ireland
}

\begin{abstract}
Automated detection of sleep apnoea from the surface ECG is a challenge with potentially significant clinical benefit. The objective of this study is to explore a possible relation between $P$-wave shape changes and the presence of Obstructive Sleep Apnoea (OSA) episodes in ECG records. Our working hypothesis is that apnoea-related phenomena (e.g., hypoxia, changes in intra-thoracic pressure) influence the depolarisation dynamics of the atria, and hence lead to detectable shape changes in the surface $P$-wave. For this purpose, a $P$-wave shape clustering algorithm is proposed. It uses the k-means method, an unsupervised classifier, coupled with a shape metric, the CISA (Corrected Integral Shape Averaging) distance, to distinguish apnoeic and non-apnoeic epochs. The method was applied to perform two-clustering on $P$ wave shapes extracted from ECG segments. The results obtained indicate that shape information from the ECG can be used in a classification approach for OSA detection. The study also raises the question of elucidating the mechanisms of how OSA may alter Pwave shape.
\end{abstract}

\section{Introduction}

Obstructive Sleep Apnoea (OSA) is a common sleep disorder with many physiological consequences, such as increased risk of cardiovascular disease, hypertension, and daytime sleepiness. It is commonly defined as the cessation of breathing during sleep [1], [2]. Usually, OSA is diagnosed through expensive hospital-based studies called polysomnograms and requires the presence of technical expertise for polysomnogram evaluation. Recently, studies were conducted, encouraged by a previous Computers in Cardiology challenge (CinC 2000), to reduce the amount of data required for detection of patients with OSA [1]. This challenge focused on the use of ECG records only. Successful approaches were developed which exploited the correlation between heart rate variation and the occurrence of OSA episodes to construct classification rules [1], [2]. However, none of these approaches significantly explored morphological changes of ECG waves related to OSA, even though such approaches could be relevant for physiological interpretation and diagnosis [3], [4]. The objective of this study is to investigate the link between P-wave shape changes and the presence of OSA. For this purpose, a Pwave shape clustering algorithm using a shape distance and centre using the CISA approach is proposed. The method is applied on extracted P-waves from ECG segments. First, a clustering, based solely on shape variation measure, is carried out to distinguish apnoeic and non-apnoeic segments labelled by polysomnogram expertise. Secondly, the same approach is employed to detect the onset and end of the OSA episodes inside some apnoeic segments. The efficacy of using P-wave shape changes for OSA detection is discussed, and some plausible mechanisms for the observed phenomenon are presented.

\section{Methods}

While the shape of components of the ECG is widely used in clinical practice to visually classify beat types, quantitative analysis of changes in shape is relatively under-utilized. We have previously developed a variety of algorithms for quantifying morphological features (shape) of the ECG signal. In general, quantitative analysis of shape is quite challenging as jitter and scaling effects mean that simple averaging of signals and Euclidean distance using yield poor estimates of shape, and shape variability. Techniques such as Integral Shape Averaging (ISA) or Dynamic Time Warping allow the true shape of a signal to be decoupled from local changes due to jitter or scaling [5]. We have also developed some novel metrics for quantifying differences between shape, such as shape distances, which allow us to compare shape across multiple beats. This can be combined with clustering algorithms, which can use shape distance to a reference shape as clustering metric. In previous work, we have used the well known k-means clustering algorithm, and a similarity criterion coupled with the ISA mean to 
identify P-wave shape changes in atrial fibrillation [3]. In that work, while the obtained clustering results were useful, the clustering condition (intra-cluster variance minimisation and inter-cluster variance maximisation) was not well satisfied since the proposed criterion respected the shape equality condition [6] but was not a distance and ISA signal was not really a gravity centre. To counteract this limitation, we have recently developed a real shape distance coupled with a shape centre [6] using the Integral Shape Averaging approach. In this paper, we will use this shape distance to determine whether the shape of the P-wave is altered by the presence of apnoea. A working hypothesis is that there will be two classes of P-wave shape- one during normal respiration, and one during apnoea. Accordingly, let us assume that:

$$
S_{i}=\tilde{S} \circ \varphi_{i}, \varphi_{i}=\psi_{i}^{-1}
$$

where $S, \tilde{S}$ and $\varphi_{i}\left(\varphi_{i}=\psi_{i}^{-1}\right)$ are respectively the normalised integral of the signal $\mathrm{x}_{\mathrm{i}}$ as defined in [6], the ISA mean and a time warping linking the two. We suppose that the time transformation $\varphi_{i}$ can be decomposed on two parts, a stochastic affine time transform $A_{t, i}$ that represents possible jitter and scale effects and a stochastic non linear time transform $\omega_{i}$ that represents the shape variation, on a constant time support, of $S_{i}$ related to $\tilde{S}$. We can write:

$$
\varphi_{i}=A_{t, i} \circ \omega_{i}
$$

where $A_{\bar{\tau}, i}(\bar{t})=\left(\bar{t}-b_{i}\right) / a_{i} \quad$ and $\quad a_{i}$ and $\quad b_{i}$ are realisations of the r.v's $A_{i}$ and $B_{i}$ with $\mathrm{E}[\mathrm{A}]=1$ and $\mathrm{E}[\mathrm{B}]=0$ respectively. Replacing eq.(1) in (2) we obtain:

$$
S_{i}=\tilde{S} \circ A_{t, i} \circ \omega_{i} \text { or } S_{i}^{-1}=\omega_{i}^{-1} \circ A_{t, i}^{-1} \circ \tilde{S}^{-1}
$$

or in another form:

$$
S_{i}^{-1}(y)=\omega_{i}^{-1}\left(a_{i} \tilde{S}^{-1}(y)+b_{i}\right), y \in[0,1]
$$

Since our shape distance proposes to measure the inverse shape fluctuation functions $\omega_{i}^{-1}$ independently from the affine time transforms, we propose to cancel the effect of $A_{\bar{t}, i}^{-1}$ before calculating our distance by estimating it from the precedent equation. For this purpose, since $A_{t, i}^{-1}$ is not directly observable we can impose that:

$$
S_{i}^{-1}(y)=a_{i}\left(\tilde{S}^{-1}(y)\right)+b_{i}+\varepsilon_{i}(y), y \in[0,1]
$$

where $\varepsilon_{i}$ is a noisy term that depends on the affine time transform $A^{-1}{ }_{\bar{t}, i}, \tilde{S}^{-1}$ and the non linearity of $\omega_{i}^{-1}$. The principal advantage of working with eq.(5) is that affine parameters become linear with $\tilde{S}_{i}^{-1}$. Then, we can easily estimate an affine transform $\hat{A}_{t, i}^{i}$ by linear regression between $S_{i}^{-1}$ and $\tilde{S}^{-1}$ and calculate for each signal a corrected signal $\hat{S}_{\hat{A}, i}^{-1}$ with the following expression using eq.(3) and (5):

with:

$$
\hat{S}_{\hat{A}, i}^{-1}=\hat{A}_{t, i} \circ S_{i}^{-1} \text { or } \quad S_{\hat{A}, i}^{-1}=\hat{\omega}_{i}^{-1} \circ \tilde{S}^{-1}
$$

$$
\hat{\omega}_{i}^{-1}=\hat{A}_{\bar{t}, i} \circ \omega_{i}^{-1} \circ A_{\bar{t}, i}^{-1}
$$

Where $\hat{\omega}_{i}^{-1}$ is an estimation of $\omega_{i}^{-1}$ obtained following the proposed method. It can be observed that the estimation of $\hat{A}_{\bar{t}, i}$ using the linear regression is suboptimal. Indeed, the non linearity represented by $\varepsilon_{i}$ for each signal can introduce an error in the estimation. As a result, $\tilde{S}^{-1}$ may be not the mean of $S_{\hat{A}, i}^{-1}$. To satisfy the gravity centre condition, we propose to recalculate a new mean from the new samples $S_{\hat{A}, i}^{-1}$ :

$$
\tilde{S}_{\hat{A}}^{-1}(y)=\frac{1}{N} \sum_{i=1}^{N} \hat{S}_{\hat{A}, i}^{-1}(y), y \in[0,1]
$$

or in another form:

$$
\tilde{S}_{\hat{A}}^{-1}(y)=\frac{1}{N} \sum_{i=1}^{N} \hat{\omega}_{i}^{-1}\left(\tilde{S}^{-1}(y)\right), y \in[0,1]
$$

and in the signal domain:

$$
\tilde{S}_{\hat{A}}=\left(\left(\tilde{S}_{\hat{A}}\right)^{-1}\right)^{\prime}
$$

We propose to name the obtained mean as Corrected Integral Shape Averaging (CISA) mean. Finally, the following CISA distance in $L^{2}[0,1]$ between samples $x_{i}$ and $x_{j}$ is given by:

$$
d_{C I S A}\left(x_{i}, x_{j}\right)=\left[\delta\left(\hat{S}_{\hat{A}, i}^{-1}(y)-\hat{S}_{\hat{A}, j}^{-1}(y)\right)^{2} d y\right]^{1 / 2}
$$

by replacing $S_{\hat{A}, i}^{-1}$ and $S_{\hat{A}, j}^{-1}$ by their value, we can rewrite eq.(10) in the following form:

$$
d_{C I S A}\left(x_{i}, x_{j}\right)=\left[f\left(\left(\hat{\omega}_{i}^{-1}-\hat{\omega}_{j}^{-1}\right)\left(\tilde{s}^{-1}(y)\right)\right)^{2} d y\right]^{1 / 2}
$$

From this last definition, we can observe that this distance is directly related to difference between two estimated inverse shape fluctuation functions $\hat{\omega}_{i}^{-1}$ and $\hat{\omega}_{j}^{-1}$ defined from the reference $\tilde{S}^{-1}$.

\section{Results}

Using the CISA mean and distance coupled with the $\mathrm{k}$ means algorithm, we proposed to develop an unsupervised shape classifier and apply it to detect OSA 
episodes.

The method was applied to first perform a "macro" clustering on P-wave shapes extracted from 93 ECG segments sampled at $128 \mathrm{~Hz}$. These segments are each two minutes in length and were acquired from five patients who suffer from OSA. For these segments, 50 are considered as normal and 43 are centered on a discrete episode of OSA. This labelling was obtained by polysomnogram analysis by an expert. For each segment, the P-waves were segmented, averaged and baseline corrected after upsampling (by a factor of five) and filtering (B-splines interpolation). For each subject, a two-class (Normal, Apnoea) clustering was performed on its corresponding segments by the proposed classifier. The clustering was done on 15 trials with random cluster centre initialisation. The selection of the final clustering was done following the combination of 3 criteria: initial inter-cluster centre shape distance maximisation, final inter-cluster and intra-cluster shape variance ratio maximisation and solution redundancy. For the five patients, the results obtained are shown in Table. 1. The method reached an overall sensitivity of $84.3 \%$ and specificity of $88 \%$. These results indicate a strong correlation between $\mathrm{P}$-wave shape changes and the occurrence of OSA.

\begin{tabular}{lccc}
\hline Patient & seg. $\mathrm{n}^{\circ}$. & Sens.(\%) & Spec. $(\%)$ \\
\hline Patient 1 & 20 & 100 & 80 \\
Patient 2 & 20 & 60 & 100 \\
Patient 3 & 20 & 90 & 70 \\
Patient 4 & 17 & 71,4 & 100 \\
patient 5 & 16 & 100 & 90 \\
& & & \\
\hline Average Scores : & 84,3 & 88 \\
\hline
\end{tabular}

Table.1: Clustering results.

A second clustering defined as "micro" clustering has also been carried out. It consists of applying the method to apnoeic segments to detect, beat by beat, the onsets and ends of the OSA episodes. For this purpose two segments are chosen. The first one is similar to those of our database. It contains one principal OSA episode centered on the segment. All the P-waves from the segment are extracted, upsampled and filtered. We can see in Figure.1 (reading from the top to the bottom) the respiration measure (ribcage) employed in the polysomnogram (sampled at $8 \mathrm{~Hz}$ ), the classification beat by beat obtained with our approach and the $\mathrm{O}_{2}$ saturation measured on the finger (sampled at $8 \mathrm{~Hz}$ ). We can observe the good fit between the beginning and the end of the respiration event, indicating the presence of an OSA
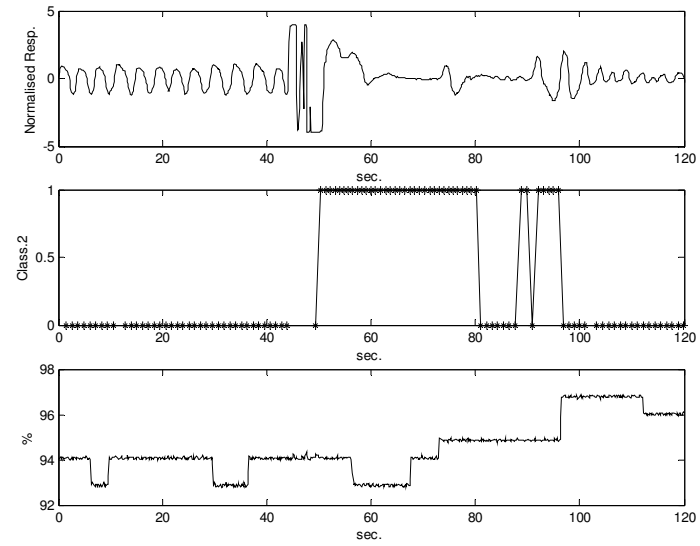

Fig.1: Respiration, CISA clustering, and $\mathrm{O}_{2}$ saturation for the first studied segment.

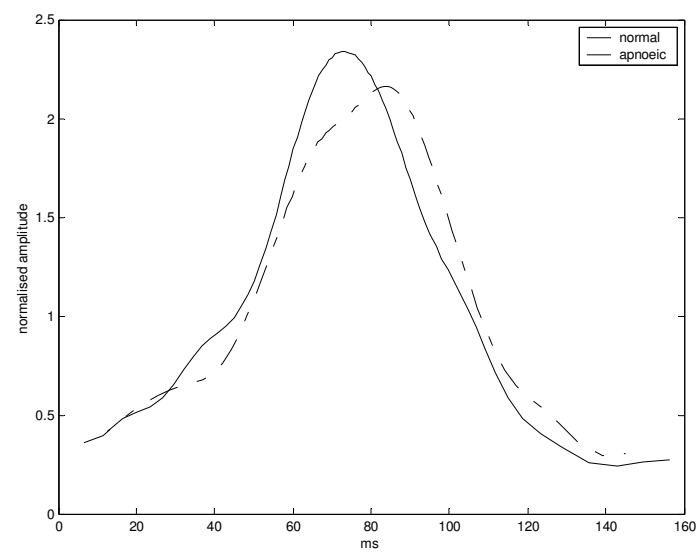

Fig.2: Apnoeic and non-apnoeic final CISA centres.
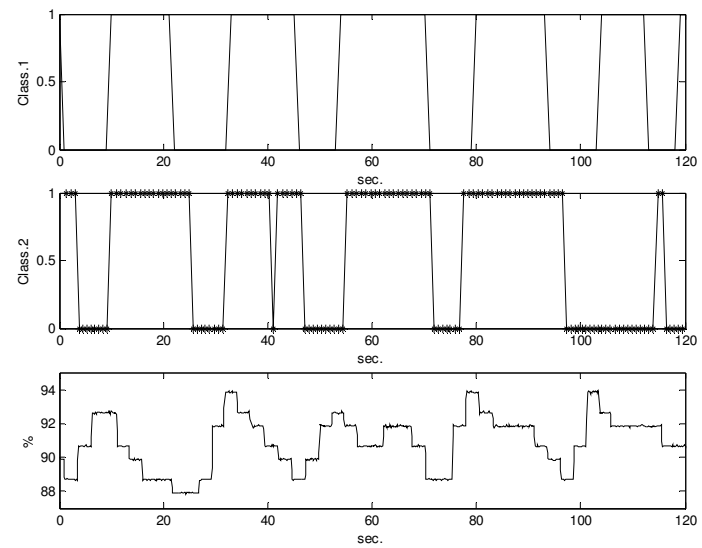

Fig.3: Expert detection, CISA clustering, and $\mathrm{O}_{2}$ saturation for the second studied segment. 
episode, and our classification. In addition, the method is sufficiently sensitive to track a small episode (after the principal one) as can be seen on the figure. In Figure.2, we illustrate the final CISA means for apnoeic and nonapnoeic P-waves inside the segment. We note a significant shape difference between the two signals. The second segment presented gives an interesting example of a rapid succession of OSA episodes, as commonly encountered in clinical practice. As can be seen in figure.3, the episodes are well detected with our approach (Classification 2) as compared with the detection (Classification 1) done by an expert viewing the full respiration signal. However an interesting difference is seen in the fifth episode, which is detected from the respiration signal but not with our method. A distinguishing feature of this episode can be seen in the corresponding $\mathrm{SpO}_{2}$ saturation signal. The first four episodes have a significant decrease in $\mathrm{SpO}_{2}$ (from $92 \sim 94 \%$ in the beginning to $88 \sim 89 \%$ in the end) indicating a significant level of hypoxia. This degree of hypoxia is not present for the fifth episode. Since our method does not detect this episode, it suggests that Pwave shape variations are strongly correlated to the degree of hypoxia during OSA episodes. In the next section, we will explore more deeply possible link between hypoxia and the observed P-wave shape changes.

\section{Discussion and Conclusions}

Through the proposed study, a strong correlation between P-wave shape changes and the occurrence of OSA episodes was clearly established. A clustering algorithm exploiting this observation was developed. It employed a CISA shape distance and centre which was recently proposed, together with the k-means algorithm. The main characteristic of the CISA approach is its ability to measure shape differences using a real distance independently of the presence of affine time and amplitude transforms. A "macro" clustering, first applied on an ECG database yielded a sensitivity of $84.3 \%$ and a specificity of $88 \%$ based solely on P-wave shape measures. A second "micro" clustering was also proposed. The results obtained demonstrated the ability to track OSA episodes using our method. In addition, a strong link between P-wave changes and hypoxia degree was observed. Results suggest the usefulness of P-wave shape changes in assessment of OSA. For future work, the study could be extended to other components of the ECG waveform, coupled with a supervised classifier. In addition, estimated affine parameters could be used as additional discriminative factors.

From a physiological point of view, our hypothesis is that hypoxia plays an important role in the occurrence of the P-wave shape changes. It has been previously shown that hypoxia disinhibits sympathetic outflow [7], which may influence atrial dynamics through localised changes in conduction velocity [4]. In fact, the P-wave shape changes observed on figure. 2 are highly similar to those observed on P-waves influenced by effort, which was presented and simulated in [4]. In exercise, similar to hypoxia, the sympathetic nervous system is further activated during effort [4]. With regard to intra-thoracic pressure changes, the effects of OSA on the left ventricle has been observed [7], but no work has been published on effects in the atria. We hope that the proposed work has enlighten the shape analysis utility for pathology detection and will encourage further research.

\section{References}

[1] Penzel $\mathrm{T}$ et al. Systematic comparison of different algorithms for apnoea detection based on electrocardiogram recordings. Med \& Biol Eng \& Comp 2002;40: 402-407.

[2] Chazal P et al. Automated Processing of the Single-Lead Electrocardiogram for the Detection of Obstructive Sleep Apnoea. IEEE Trans Biomed Eng 2003;50:686-696.

[3] Boudaoud S, Rix H, Blanc JJ, Cornilly JC, Meste O. Integrated Shape Averaging Applied To AF Detection. Computers in Cardiology 2003; 30:125-128.

[4] Boudaoud S, Meste O, Rix H. Curve Registration for Study of P-wave Morphing during Exercise. Computers in Cardiology 2004; 31:125-128.

[5] Boudaoud S, Rix H, Meste O. Integral Shape Averaging and Structural Average Estimation: A Comparative Study. IEEE Trans Sig Proc 2005; 10.

[6] Boudaoud S, Rix H, Meste O. Providing Sample Shape Statistics with FCA and ISA Approaches. Stat \& Sig Proc IEEE Workshop SSP'05, Bordeaux, Jul. 22-25, 2005, CDROM.

[7] Leung RS, Bradley TD. Sleep Apnea and Cardiovascular Disease. Am J Respir Crit Care Med 2001; 164:2147-2165.

Address for correspondence.

Sofiane Boudaoud

Lab. I3S, UNSA-CNRS, 2000, route des Lucioles. BP 121 06903 Sophia Antipolis Cedex , France

tel. / fax: +3304929651 04/ +330492965155

E-mail: Boudaoud@i3s.unice.fr 